\title{
Anesthesiology and Critical \\ Care Response to COVID-19 \\ in Resource-Limited Settings
}

Experiences from Nepal

Gentle S. Shrestha, MD, EDIC, FCCP, FNCS ${ }^{a}$ *, Ritesh Lamsal, MD, DM ${ }^{a}$, Pradip Tiwari, MD ${ }^{b}$, Subhash P. Acharya, MD, FCCP ${ }^{a}$

\section{KEYWORDS}

- Anesthesiology • Critical care • COVID-19 • Nepal • Resource-limited

\section{KEY POINTS}

- Most of the world's population does not have access to safe and affordable surgical and anesthesia care.

- Anesthesia and critical care delivery systems in resource-limited settings grappling to manage patients have been overwhelmed by the current COVID-19 pandemic.

- Evidence derived from high-income countries and international guidelines on COVID-19 cannot be easily implemented or may not be applicable in resource-limited settings.

- Low- and middle-income countries must perform high-quality indigenous research, use innovative measures, determine minimal standards of care, and focus on attainable clinical goals.

\section{INTRODUCTION}

As the human population continues to rise, the global burden of surgical procedures and the need for anesthesia and critical care is increasing. According to the World Health Organization, the global burden of surgeries was more than 310 million in 2012 , with an increase of nearly $40 \%$ from a similar estimate in $2004 .^{1}$ A recent report highlighted that nearly 5 billion people do not have access to safe, affordable surgical and anesthesia care, and one-third of the global burden of disease requires surgery and anesthesia management. ${ }^{2}$ However, only a small number of surgeries are carried out in low- and middle-income countries (LMICs), mainly because of the lack of

\footnotetext{
a Department of Anaesthesiology, Tribhuvan University Teaching Hospital, Maharajgunj Road, PO Box: 1524, Maharajgunj, Kathmandu 44600, Nepal; ${ }^{b}$ Department of Critical Care, Norvic International Hospital, PO Box: 14126, Thapathali, Kathmandu 44617, Nepal

* Corresponding author.

E-mail address: gentlesunder@hotmail.com
} 
resources and personnel. The disparity in the distribution of health care among countries will probably widen in the near future, because LMICs have a higher population growth compared with high-income countries.

The pandemic of COVID-19 is evolving. The spectrum of COVID-19 varies from asymptomatic cases to severe disease requiring intensive care admission and multiorgan support. Nearly $80 \%$ of COVID-19 patients experience mild-to-moderate symptoms and around $5 \%$ of cases require intensive care unit (ICU) admission. ${ }^{3}$ However, the distribution and severity of COVID-19 are substantially dissimilar in different parts of the world. The mortality rate of cases admitted to the ICU is between $23.4 \%$ and $33 \%$ and the average length of ICU admission is 10.8 days. ${ }^{4}$ The mortality rate among patients receiving mechanical ventilation is $43 \%$ to $67 \%$, and it is close to $70 \%$ among patients older than 60 years. ${ }^{5}$

Around the world, providing safe anesthesia and critical care services has been a major challenge during the pandemic. This problem is more pronounced in LMICs. Anesthesia and critical care services are easily overwhelmed in LMICs because of the dense population with a high rate of infection, limited testing capacity and weak contact-tracing, inadequate hospital and critical care beds, inadequate anesthesiologists and intensivists, weak health care infrastructure, and limited access to therapeutics and personal protective equipment (PPE). Most LMICs responded to the COVID-19 pandemic with various public health measures, such as strict lockdown, social distancing, and universal masking. The measures were initially effective in reducing the rapid spread of the infection, but most of these stringent measures have since been relaxed in many countries. The limitations of treating critically ill patients, even in high-income countries, have been abundantly discussed in academic and public forums in the current pandemic. ${ }^{6}$

\section{Epidemiology of COVID-19 in Nepal}

Nepal is one of the poorest countries of the world with a nominal gross domestic product per capita of only around US $\$ 1070 .^{7}$ The first case of COVID-19 in Nepal was diagnosed in January 2020, through testing done in Hong Kong, because of the unavailability of the reverse-transcriptase polymerase chain reaction test in Nepal. ${ }^{8}$ Because Nepal has limited health care capacity, including of intensive care beds, a strict national lockdown with social distancing measures was imposed for months, from March to July 2020. However, since easing the national lockdown, Nepal has seen a surge of COVID-19 cases and deaths. ${ }^{9}$ As of November 8,2020 , there are more than 194,000 confirmed cases of COVID-19, with 1108 deaths in Nepal. ${ }^{9}$ The future course and outcome of the pandemic are impossible to predict because the numbers of new infections and deaths continue to increase at an alarming rate.

\section{DISCUSSION}

The ongoing pandemic has severely affected all sectors of Nepal's health care delivery system. Anesthesia and critical care services are hard hit, because sparse health resources and public spending have been diverted to the containment of the infection, and the management of COVID-19 patients. In Nepal, the first ICU was established in 1973, and this was the only well-equipped ICU in the country for close to 20 years. ${ }^{10}$ As of April 15, 2020, Nepal had only 1595 ICU beds for a population of nearly 30 million, and only around $850 \mathrm{ICU}$ ventilators. ${ }^{11}$ The intensive care facilities were already stretched before the pandemic, and with the rapid surge of patients requiring ICU admission, Nepal is currently experiencing a critical shortage of ICU beds. 
Lately, there is a concerted effort by public and private health institutions to develop more facilities for the management of COVID-19 patients. Several existing postoperative wards and other high-dependency units have been converted into improvised critical care units. However, ICU equipment and trained personnel are scarce. ${ }^{12}$ It is difficult to procure essential ICU equipment and personal protective devices because medical manufacturing facilities are nonexistent in the country. The government, and private institutions, have already spent enormous sums developing new makeshift ICUs and procuring consumables; it is doubtful whether most of these institutions can fund the additional cost of running optimal services in the operating rooms (ORs) and ICUs.

\section{Early Response to the Pandemic}

Nepal, like most resource-limited countries, was unprepared to face the rapid upsurge of a highly infectious disease. There was only one dedicated tertiary-level infectious disease hospital in the entire country before the current pandemic. There is also huge variability in the distribution of health care across the country, with poor health infrastructure outside a few big cities. Initially, the Government of Nepal segregated all government, and some private hospitals, into either COVIDdedicated hospital or non-COVID hospital. However, with the rapid increase in the number of patients, and limited health centers that could cater to such patients, the COVID-19 hospitals were soon overwhelmed and it was no longer possible to maintain the segregation. Currently, all hospitals in the country manage patients, irrespective of COVID-19, unless patients have to be referred to a higher center for specialized care.

After the initial lockdown, all elective surgeries were stopped as the focus shifted to managing patients with COVID-19. In many hospitals, this policy continues to date with surgical departments only treating patients with urgent or emergency conditions. With limited resources, anesthesiologists were forced to improvise to decrease the risk of contagion. Many anesthesiologists and intensivists started using videolaryngoscopes for the first time. Several types of barrier protective devices, such as transparent aerosol boxes and plastic sheets, were used to cover the patient's head during aerosol-generating procedures (eg, endotracheal intubation, airway suctioning, and extubation). Many standard recommendations that were not cost-prohibitive, such as the use of high-efficiency viral filters and minimizing aerosol-generating procedures, were followed.

\section{CURRENT CHALLENGES TO ANESTHESIOLOGY AND CRITICAL CARE SERVICE DELIVERY IN NEPAL}

The health care system in Nepal is mixed, with public health facilities and private hospitals providing health services. Public health institutions are controlled by the government and organized at central, provincial, and local levels. The infrastructure in most of the government-funded hospitals outside the large cities is weak, and often strained even at normal times. ${ }^{13}$ Most of the ICU beds are within private health care facilities. As in many LMICs, health care facilities are concentrated in cities, but most people live in rural areas, creating inequality in access to health care. Similarly, COVID-19 testing facilities are only available in big cities. In private hospitals, surgical and intensive care costs are expensive and not readily affordable by most of the population. There are added financial constraints because the health insurance system is almost nonexistent in the country. 


\section{Infrastructure and Personnel}

The capacity for critical care delivery in LMICs is limited. Most LMICs in Asia have fewer than 3 ICU beds per 100,000 population; Nepal has 2.8 ICU beds per 100,000 population, whereas Germany has 29.4 ICU beds, and the United States has 34.7 per 100,000 population. ${ }^{14,15}$ Most of the ORs and ICUs do not have the provision of a negative-pressure system. Even in other Asian countries, less than $40 \%$ of the ICUs have the provision of negative-pressure rooms. ${ }^{16} \mathrm{~A}$ central monitoring system and invasive and advanced monitors are limited to few tertiary-care centers.

A recent cross-sectional study of all COVID-care hospitals in Nepal found a lack of most basic equipment in the ICU. ${ }^{17}$ Only around $63 \%$ of the hospitals had a defibrillator, and only $26.7 \%$ of hospitals could process arterial blood gas samples. ${ }^{17}$ Nearly $47 \%$ of the hospitals did not have a central pipeline supply for oxygen. There are no published data about the availability of other essential OR and ICU equipment, such as infusion pumps, noninvasive ventilation, high-flow nasal cannula, and hospital beds equipped with oxygen supply. There is also a pressing need for more health care workers. Nepal has only 1 doctor per 1000 people, which is well lower than the World Health Organization's recommendation of 2.3 doctors per 1000 population. ${ }^{18}$

Most of the ICUs in the country are staffed by anesthesiologists and internists. There are few physicians with additional training in critical care. There are only a handful of critical care nurses. Other ICU workers, such as respiratory therapists and clinical pharmacists, are not found in most hospitals. Health care institutions in LMICs were already struggling with the lack of trained workers before the pandemic. With the surge of COVID-19, many health care workers have also been infected, significantly overburdening the existing hospital workforce. This may worsen outcomes for patients, because adequate staffing is crucial to maintain proper patient care. ${ }^{19}$ Proper strategies to support and manage infected workers also seem to be lacking. In many hospitals, health care staff is now asked to work simultaneously in COVID-19 and nonCOVID-19 areas of the hospital. Even infected patients have been shifted to nondesignated areas of the hospital because COVID-19-dedicated areas are overflooded with infected patients, increasing the risk of spreading the infection.

There is also a scarcity of essential anesthesia and critical care equipment for COVID-19 patients. For instance, ultrasonography and other radiologic devices may not be available in COVID-19-designated areas of the hospital. There may also be difficulty in obtaining radiographs and echocardiography tests because of the logistical difficulties in sharing the same facilities and equipment between infected and noninfected patients. This not only adds to the difficulty for the anesthesiologists and intensivists, but severely compromised patient care.

\section{Infection Control Measures}

Health care workers are at risk of contracting COVID-19 because of frequent contact with infected patients. The overall proportion of health care workers infected with COVID-19 is around $10 \% .^{20}$ This proportion is approximately $18 \%$ in the United States, $4 \%$ in China, and $9 \%$ in Italy. ${ }^{20}$ The availability of good-quality PPE kits, proper use of PPE, and infection control training programs are key to reduce the incidence of health care-associated infection. The World Health Organization has recommended the use of gloves, masks, goggles or face shields, and long-sleeved gowns with N95 respirators for aerosol-generating medical procedures. In many LMICs, the supply and availability of PPE are inadequate. The surgical masks and N95 masks are reused in most hospitals in Nepal. Because certified respirators are in short supply, the fit-test is rarely carried out. The government has encouraged local production of 
PPE, but quality control and surveillance are limited, endangering the safety of health care workers. Face shields and goggles are shared after disinfection. The use of reusable elastomeric respirators and reusable powered air-purifying respirators are alternatives, but their procurement has been difficult.

An infection prevention and control (IPC) program, with a dedicated trained team at the health care facility, is recommended in all hospitals. IPC measures, if implemented appropriately, protect patients, health care workers, and visitors. In an international survey, only $11 \%$ of respondents from low-income countries had consistent access to respiratory equipment, $12 \%$ to isolation gowns, and $4 \%$ to negative-pressure rooms or personnel training in IPC. ${ }^{21}$ Inadequate access to IPC equipment and training can lead to major COVID-19 outbreaks in hospitals and institutional isolation centers. Most hospitals in Nepal do not have the provision of IPC programs. Similarly, because of constraints of space and resources, separate areas for proper donning and doffing of PPE are not available in most ORs and ICUs, which significantly adds to the risk of viral contamination.

\section{Quality Care}

The delivery of quality care during any pandemic is challenging because crucial scientific information is always evolving. Therapeutic options may also be uncertain. A recent study found a significant disparity in the number of COVID-19-related studies and publications between high-income countries and LMICs. ${ }^{21}$ The protocols, guidelines, and therapeutic options for COVID-19 are mostly formulated based on studies and experiences of high-income countries. However, such guidelines may not necessarily address the unique problems of resource-limited settings. Some of the standard guidelines may not even be feasible in most ORs and ICUs in Nepal and other resource-limited countries because of resource-constraints, and significant variability in the structure and composition of the workforce. Data-driven quality improvement programs in high-income countries have helped to identify gaps in health care service delivery, and design "improvement approaches." There is a glaring lack of such programs in resource-limited settings.

\section{Future Directions}

In the current pandemic, there is a significant overload of clinical information, but without the accompanying high-quality clinical research. There is a paucity of unifying clinical guidelines and a lot of uncertainty about the best treatment options, resulting in dissimilar treatment decisions between patients. There is an urgent need for research focused on LMICs, because the scientific evidence derived from high-income countries may not be accurate when applied in a different setting. For instance, the population in LMICs may be younger than in the western world and may have fewer risk factors for developing severe COVID-19, such as obesity. Most hospitals in LMICs may also not be able to meet the standards recommended by international guidelines. An option may be for international bodies to develop or propose the minimal safety standards for anesthesia and critical care services in LMICs, considering the available resources, without compromising the safety of patients and health care workers. For example, dexamethasone is readily available and can be administered in most patients. It can be prioritized over expensive therapeutics of questionable benefits that are not easily available in low-resource settings, such as newer antivirals, immunomodulators, and convalescent plasma therapy.

The current and previous pandemics have pushed innovation. These innovations are extremely handy when resources are limited. Experiences from the SARS epidemic in Hong Kong suggested that when negative-pressure isolation rooms are 


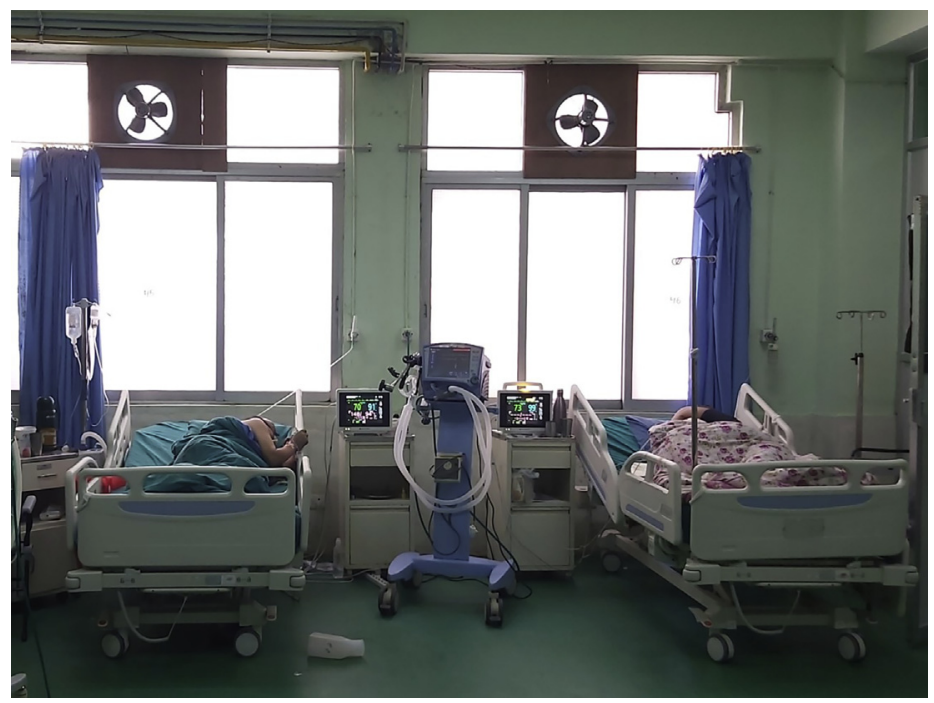

Fig. 1. Postanesthesia care unit is converted into COVID ICU. Because of lack of negativepressure system, industrial exhaust fans were installed to minimize the viral load.

not available, normal rooms can be converted to makeshift negative-pressure rooms by the use of industrial exhaust fans. ${ }^{22}$ In our center, we converted the postoperative care unit into a COVID ICU. We applied industrial exhaust fans over each patient bed, attempting to create negative-pressure systems and to minimize the viral load (Fig. 1). Similarly, several cost-effective improvised videolaryngoscopes, PPE kits, and ventilators have been designed recently. ${ }^{23-27}$ However, these improvised surrogates should be tested for safety and effectiveness before generalized use. Because the population of the LMICs is young with possibly fewer risk factors, resourceconstrained countries may still achieve acceptable clinical outcomes by determining the minimal standards of care, and doing the basics right.

\section{SUMMARY}

In resource-limited settings, it is often not possible or practicable to meet the clinical targets stipulated in international guidelines. This difficulty is more pronounced in the ongoing pandemic because the optimal use of scarce resources is crucial. It is useful for health care delivery systems in resource-limited settings to promote pragmatic innovative measures, adapt international guidelines to suit local needs, focus on diagnostic and therapeutic measures that are easily accessible and less resourceintensive, and promote native research activities. It is equally important to shift the focus beyond ICU admissions, advanced monitoring, and therapeutic requirements to other important aspects of treating COVID-19 patients where there are better chances of alleviating suffering and providing equitable care. Integrating hospice and palliative care into the health care system, which is strikingly deficient in LMICs, will also go a long way in providing dignified end-of-life care during the COVID-19 pandemic.

\section{DISCLOSURE}

The authors have nothing to disclose. 


\section{REFERENCES}

1. Weiser TG, Haynes AB, Molina G, et al. Size and distribution of the global volume of surgery in 2012. Bull World Health Organ 2016;94:201-209f.

2. Alkire BC, Raykar NP, Shrime MG, et al. Global access to surgical care: a modelling study. Lancet Glob Health 2015;3e:316-23.

3. Wu Z, McGoogan JM. Characteristics of and important lessons from the coronavirus disease 2019 (COVID-19) outbreak in China: summary of a report of 72314 cases from the Chinese Center for Disease Control and Prevention. JAMA 2020; 323:1239-42.

4. Tan E, Song J, Deane AM, et al. Global impact of COVID-19 infection requiring admission to the intensive care unit: a systematic review and meta-analysis. Chest 2020;159(2):524-36.

5. Wunsch $\mathrm{H}$. Mechanical ventilation in COVID-19: interpreting the current epidemiology. Am J Respir Crit Care Med 2020;202:1-4.

6. Herreros B, Gella P, Real de Asua D. Triage during the COVID-19 epidemic in Spain: better and worse ethical arguments. J Med Ethics 2020;464:55-8.

7. GDP per capita (current US\$). World Bank national accounts data, and OECD National Accounts data files. Available at: https://data.worldbank.org/indicator/ NY.GDP.PCAP.CD. Accessed November 1, 2020.

8. Bastola A, Sah R, Rodriguez-Morales AJ, et al. The first 2019 novel coronavirus case in Nepal. Lancet Infect Dis 2020;202:79-80.

9. Covid-19 cases map representation. Ministry of health and population Nepal. Available at: https://covid19.mohp.gov.np. Accessed November 8, 2020.

10. Acharya SP. Critical care medicine in Nepal: where are we? Int Health 2013; 59:2-5.

11. Government of Nepal: Ministry of Health and Population. Health Sector Emergency Response Plan COVID-19 Pandemic. 2020. Available at: https://www. who.int/docs/default-source/nepal-documents/novel-coronavirus/health-sectoremergency-response-plan-covid-19-endorsed-may-2020.pdf?sfvrsn=ef831f44_ 2. Accessed November 5, 2020.

12. Shrestha GS, Paneru HR, Acharya SP, et al. Preparedness for coronavirus disease in hospitals of Nepal: a nationwide survey. JNMA J Nepal Med Assoc 2020;582:48-51.

13. Neupane HC, Gauli B, Adhikari S, et al. Contextualizing critical care medicine in the face of COVID-19 pandemic. JNMA J Nepal Med Assoc 2020;584:47-52.

14. Phua J, Faruq MO, Kulkarni AP, et al. Critical care bed capacity in Asian countries and regions. Crit Care Med 2020;486:54-62.

15. Ma X, Vervoort D. Critical care capacity during the COVID-19 pandemic: global availability of intensive care beds. J Crit Care 2020;589:6-7.

16. Arabi YM, Phua J, Koh Y, et al. Structure, organization, and delivery of critical care in Asian ICUs. Crit Care Med 2016;44:e940-8.

17. Assessment of health-related country preparedness and readiness of Nepal for responding to COVID-19 pandemic. Preparedness and readiness of government of Nepal designated COVID hospitals 2020. Available at: http://nhrc.gov.np/wpcontent/uploads/2020/06/Fact-sheet-Preparedness-and-Readiness-of-Governmentof-Nepal-Designated-COVID-Hospitals.pdf. Accessed October 25, 2020.

18. The World Bank. Physicians (per 1,000 people) - Nepal. Available at: https://data. worldbank.org/indicator/SH.MED.PHYS.ZS?locations=NP. Accessed October 19, 2020.

19. Remuzzi A, Remuzzi G. COVID-19 and Italy: what next? Lancet 2020;395:1225-8. 
20. Sahu AK, Amrithanand VT, Mathew R, et al. COVID-19 in health care workers: a systematic review and meta-analysis. Am J Emerg Med 2020;38:1727-31.

21. Usuzaki T, Chiba S, Shimoyama M, et al. A disparity in the number of studies related to COVID-19 and SARS-CoV-2 between low- and middle-income countries and high-income countries. Int Health 2020. https://doi.org/10.1093/ inthealth/ihaa088.

22. Gomersall CD, Tai DY, Loo S, et al. Expanding ICU facilities in an epidemic: recommendations based on experience from the SARS epidemic in Hong Kong and Singapore. Intensive Care Med 2006;32:1004-13.

23. Hamal PK, Chaurasia RB, Pokhrel N, et al. An affordable videolaryngoscope for use during the COVID-19 pandemic. Lancet Glob Health 2020;8:e893-4.

24. Saoraya J, Musikatavorn K, Sereeyotin J. Low-cost videolaryngoscope in response to COVID-19 pandemic. West J Emerg Med 2020;21:817-8.

25. Chien LC, Beÿ CK, Koenig KL. A positive-pressure environment disposable shield (PEDS) for COVID-19 health care worker protection. Prehosp Disaster Med 2020;35:434-7.

26. Fang Z, Li Al, Wang $\mathrm{H}$, et al. AmbuBox: a fast-deployable low-cost ventilator for COVID-19 emergent care. SLAS Technol 2020. https://doi.org/10.1177/ 2472630320953801. 20202472630320953801.

27. Jardim-Neto AC, Perlman CE. A low-cost off-the-shelf pressure-controlled mechanical ventilator for a mass respiratory failure scenario. Br J Anaesth 2020; 125:e438-40. 